\title{
VIDEOJUEGOS EN EL APRENDIZAJE: \\ OPORTUNIDADES Y DESAFÍOS
}

VIDEOGAMES IN LEARNING:

Opportunities and challenges

\author{
Stella Massa \\ (Grupo de Investigación en Tecnologías \\ Interactivas, UNMdP)
}

\begin{abstract}
RESUMEN
En este trabajo se aborda la problemática y desafíos que se presentan en la generación de ambientes de aprendizaje que incluyan las Tecnologías de la información y de la comunicación.

En la actualidad se han desarrollado medios innovadores como las Tecnologías emergentes en las que se incluyen los videojuegos. Los videojuegos son una de las fuentes de entretenimiento especialmente para los jóvenes. Ese potencial puede explotarse en los procesos de instrucción en la medida que conozcamos más de sus atributos y de los mecanismos de incorporación en el aula. Con este objetivo se indagan y describen las características y oportunidades que ofrece el videojuego como dispositivo cultural y en particular educativo.
\end{abstract}

Palabras Clave: videojuegos cultura, educación

\section{ABSTRACT}

This paper addresses the problems and challenges that arise in the generation of learning environments that include Information and communication technologies.

Innovative media have nowadays been developed, such as emerging technologies that include video games

Video games are one of the main sources of entertainment, especially for young people. That potential can be exploited in the instructional processes as far as we know more about their attributes and the mechanisms of incorporation in the classroom. Keeping in mind this objective, the characteristics and opportunities offered by videogames as a cultural, and in this particular, as an educational device, are investigated and described.

Keywords: videogames, culture, education.

\section{Introducción}

La inclusión de las Tecnologías de la información y de la comunicación (TIC) en ambientes de aprendizaje requiere de un abordaje no sólo tecnológico y lo pedagógico. Es así que se plantea un debate acerca de las prácticas docentes y discentes atravesadas por la tecnología.

En ese sentido, Medina (2001) señala que las decisiones metodológicas de cambio en ambientes de aprendizaje deberían sustentarse en principios como los siguientes: transferencia, problematización de lo aprendido o a aprender, prospectiva, toma de decisiones, complejidad, autenticidad, realismo analógico, incertidumbre, integración del saber y hacer y multiculturalismo. 
Del mismo modo, el cambio educativo, según Fullan (2007), es un proceso dinámico que implica la interacción de variables en el tiempo. El autor agrupa los factores necesarios para implementar transformaciones en educación en tres grandes categorías: en primer lugar, las características de la innovación; en segundo lugar, las características locales tales como ubicación, nexos geográficos, gestión administrativa, autoridades y/o directriz de sectores y, finalmente, las características externas, relacionadas con las políticas gubernamentales o las iniciativas apoyadas por agencias internacionales.

En la actualidad la tecnología ha desarrollado mejores medios innovadores para llegar a mayor cantidad de personas, las denominadas "Tecnologías emergentes". Ramírez (2014) las califica como nuevas tecnologías disruptivas e innovaciones basadas en la ciencia con la capacidad de crear una industria nueva o la de transformar una existente. Comprenden las telecomunicaciones, los dispositivos móviles, la realidad aumentada, los videojuegos, la gamificación, el m-learning, el flipped classroom (clases invertidas) y los cursos masivos en línea (MOOC), entre otros.

En la última década, los investigadores han difundido el uso de juegos de computadora con el propósito de enseñar y aprender.

Considerado sólo un medio de ocio por muchos, el videojuego ofrece un potencial que radica en reconocer sus características positivas para incorporarlas en los procesos educativos.

En este artículo se presentan las problemáticas de la inclusión de las TIC en las aulas desde una visión innovadora y se focaliza en el caso particular del videojuego.

\section{El Videojuego: un dispositivo cultural}

Es insoslayable reconocer que los videojuegos se han convertido en una de las fuentes de entretenimiento principales de buena parte de la población. Además, a lo largo de sus cinco décadas de existencia, se han posicionado como una de las expresiones de creatividad humana más relevantes de la cultura contemporánea (Aranda y Sánchez Navarro, 2009).

El videojuego se perfila como la industria cultural dominante en este nuevo siglo. La riqueza cultural del juego y en particular del videojuego se debe a que se comportan como "fábricas de signos" (Aarseth, 1997).

Frasca (2009) afirma que a diferencia de los signos "tradicionales" que tienen varias interpretaciones posibles, los signos en el juego tienen además múltiples manipulaciones posibles: es de por sí polisémico y abierto a la interpretación. El estado del juego puede además ser modificado por los jugadores, de acuerdo a reglas preestablecidas. Este mismo autor insiste en que esta manipulación es claramente física y no metafórica: la obra se modifica formalmente al ser manipulada (y por ende, también su significado).

Los jóvenes de hoy son los principales consumidores de los videojuegos. Las características de ese grupo etario han sido estudiadas por varios investigadores. Tapscott (1998) indica que la "generación Internet" aprende, juega, se comunica en forma muy distinta a la de sus padres. Beck y Wade afirman que la "generación de jugador" tiene sistemáticamente diferentes formas de trabajar que son la consecuencia de un factor central: crecer con los videojuegos (Beck y Wade, 2004, 2006).

Otra denominación es la de Millennians. Este término fue utilizado por primera vez por los historiadores generacionales y sociólogos Howe y Strauss (2000) en su ensayo "Millennials Rising: The Next Great Generation". En resumen, los Millennials son la primera generación que crece rodeada de medios digitales, y la mayoría de sus actividades están relacionadas con la comunicación peer-topeer y la gestión del conocimiento, en el sentido más amplio, están mediadas por estas tecnologías.

Por otra parte, los videojuegos, como producto cultural, generan en los jugadores ciertos estados emocionales que pueden aprovecharse para el desarrollo de competencias. 
En tal sentido, la diversión es uno de los aspectos del videojuego que invita a jugar. Koster (2013) define la diversión como un efecto de aprender algo nuevo, algo que obtenemos o ganamos. Ese sentimiento de diversión es un mecanismo de feedback positivo que nos impulsa a repetir la actividad una y otra vez.

Aunque, coincidiendo con Scolari (2013), Pérez Latorre (2012), Frasca (2009) y García Gigante (2009), en realidad, los videojuegos son mucho más que un simple divertimento, ya que son parte importante de nuestra cultura al igual que sucede con otros medios como la televisión, el video, la música, la radio o los libros y deben analizarse desde esta perspectiva.

Existen otros factores que condicionan su adhesión o engagement, tales como las demandas sociales, los elementos emocionales y las etapas cognitivas; las motivaciones (social, emocional e intelectual); y, los atributos del juego/género (juego colaborativo, demandas y retos) (Sherry, 2013), pues pueden aumentar el interés y las expectativas ante la experiencia lúdica (Boyle, et al. 2016).

Csikszentmihalyi (1990) define que la integración del trabajo y el juego determinan el estado psicológico que ha denominado estado flow. Es un estado de ánimo caracterizado por la concentración enfocada y elevado disfrute durante las actividades intrínsecamente interesantes (Shernoff, Csikszentmihalyi, Schneider \& Shernoff, 2003).

Csikszentmihalyi (1990), distingue ocho elementos importantes del estado flow comunes en la mayoría de las personas durante una experiencia óptima:

- una tarea difícil pero que se puede resolver;

- los objetivos son claros, no hay ambigüedades;

- retroalimentación inmediata de la tarea;

- participación profunda, pero sin esfuerzo que elimina de la conciencia las frustraciones y preocupaciones de la vida cotidiana;

- sentido de control sobre nuestras acciones;

- no hay preocupación por sí mismo;

- hay libertad completa de concentrarse en la tarea y

- alteración del paso del tiempo.

La clave de los aportes de Csikszentmihalyi radica que para generar una "experiencia óptima" o estado flow se debe establecer una tarea en los videojuegos que suponga un "reto para el individuo", quien debe tener una predisposición hacia tareas que requieran el uso de habilidades que sólo se alcanzan después de conseguir un nivel de experiencia.

\section{Videojuego como dispositivo educativo}

El modelo enciclopedista con el que la escuela fue creada y que estudiaron la gran mayoría de los docentes, por lo menos en América latina, ha recibido numerosas críticas. Investigadores de la educación y responsables de las políticas educativas reconocen que la perspectiva centenaria del contenido que se trabaja en la escuela está lejos de lo que una persona requiere para desarrollarse en plenitud y para incorporarse a la sociedad de la que forma parte (Díaz-Barriga, 2014).

En el informe Horizont (2016) se analizan las tendencias claves y desafíos futuros en educación con TIC. En dicho informe, como tendencias a corto plazo, señalan el rediseño de los espacios de aprendizaje que incluyan actividades cada vez más prácticas y de mayor inmersión, así como repensar las escuelas trabajando al ritmo de las demandas a la fuerza laboral del siglo XXI, equipando a los estudiantes con las habilidades requeridas en el futuro.

Aunque los videojuegos proporcionan un entorno de aprendizaje rico y complejo, es necesario modificar las estrategias educativas para poder integrarlos de una forma coherente y adecuada (Gros, 2009). 
Tal como señala Valderrama (2012), el docente que desea usar estas herramientas en sus clases debe explorar el universo que ofrecen los videojuegos con una mentalidad abierta. Esto despertará su creatividad para que pueda ver aplicaciones prácticas en su asignatura.

De acuerdo con Gros (2009), el videojuego puede ser el disparador para la aproximación de un tema, el interés del grupo, un problema o parte de uno de los objetivos planteados. Entonces se convierte en una herramienta didáctica que da paso a la exploración y la investigación por parte de los estudiantes.

Sin embargo, tal como señala Padilla Zea (2011), todavía hoy, cuando colocamos la palabra educativo junto a videojuego, en nuestro subconsciente algo nos dice que no va a ser divertido. Esto se debe a que, en realidad, la mayoría de los juegos que se enmarcan en esta categoría tienen una fuerte intención educativa y dejan a un lado distintos aspectos relacionados con las características presentes en los videojuegos comerciales.

Gee (2003) destaca la capacidad de los ambientes de juego para "situar el significado en el espacio multimodal a través de experiencias encarnadas para resolver problemas y reflexionar. "(Gee, pág. 48). Los jugadores adoptan las identidades de "científico", "historiador", "arquitecto", etc.

Steiner et al. (2015) sostienen que, aunque hay un creciente cuerpo de evidencia sobre la eficacia de los videojuegos para el aprendizaje, la evaluación es a menudo mal diseñada, incompleta, sesgada, si no totalmente ausente. Las evaluaciones bien diseñadas que demuestren el efecto educativo pueden fomentar una adopción más amplia por parte de las instituciones educativas y los proveedores de capacitación y apoyar el desarrollo de la industria de los Serious Games.

En esa línea, Garris, Ahlers y Driskell (2002) definieron un modelo que identifica las características o aspectos motivadores de los videojuegos y su relación con los logros del aprendizaje.

Hay un modelo tácito de aprendizaje que es inherente en la mayoría de los estudios de videojuegos en educación. En primer lugar, el objetivo es diseñar una secuencia didáctica que incorpore ciertas características de los videojuegos. En segundo lugar, estas características desencadenan un ciclo que incluye juicios o reacciones de los usuarios tales como placer o interés, comportamientos de los usuarios como mayor persistencia o tiempo en la tarea, y retroalimentación adicional del sistema. En la medida en que tenemos éxito en equilibrar el contenido educativo con las características del videojuego, este ciclo se traduce en juego recurrente y motivado. Por último, este compromiso en el juego conduce a la consecución de los objetivos de formación y los resultados específicos de aprendizaje. Este modelo incluye los siguientes elementos:

- Contenidos educativos

- Dimensiones del juego (fantasía, reglas y metas, estímulos sensoriales, desafío, misterio y control).

- Ciclo del juego:

- Juicios del jugador (interés, disfrute, participación de la tarea y confianza).

- Comportamiento del jugador (participación sostenida, esfuerzo, concentración, persistencia y reenganche con el juego).

- Retroalimentación del sistema.

- Resultados de aprendizaje (habilidades, aprendizaje cognitivo y afectivo).

La importancia de este modelo radica en que si nos proponemos en incorporar las características propias de los videojuegos que juegan los estudiantes esto puede desencadenar un ciclo de juego, un ciclo repetitivo que caracteriza el compromiso que muestran los jugadores en los videojuegos. En la medida en que se equilibran las características del juego con el contenido educativo en forma apropiada podremos aprovechar estas fuerzas motivacionales para lograr los resultados de aprendizaje deseados. 
Sin embargo, a pesar del potencial de los videojuegos en términos de interactividad, inmersión y compromiso, todavía hay mucho trabajo por hacer para comprender mejor cómo diseñar, administrar y evaluar videojuegos en diferentes contextos y logros de aprendizaje (Alvarez \& Michaud, 2008; Ulicsac, 2010; de Freitas y Liarokapis, 2011).

\section{Serious Games: educación con tecnología}

Los Serious Games son aplicaciones interactivas creadas con una intencionalidad educativa, que proponen la explotación de la diversión e inmersión como experiencia del jugador. Al hablar de Serious Games queremos decir que el objetivo del videojuego no es entretener al jugador, lo que sería un valor añadido, sino utilizar la calidad entretenida para la formación, la educación, la salud, la política pública y objetivos estratégicos de comunicación (Zyda, 2005).

Los Serious Games se describen en términos de interactividad (Prensky, 2001; Vogel et al., 2006), basado en un conjunto de reglas acordadas y restricciones (Garris, Ahlers y Driskell, 2002) y dirigida hacia una meta que a menudo se establece por un desafío (Malone, 1981).

Investigaciones como las de Foreman (2004), Squire (2006), Yang (2012), Huang, Johnson \& Han, (2013), señalan que el carácter motivador de estos videojuegos es un factor clave para alcanzar los objetivos educativos de los mismos.

Desde esta posición, la elección del videojuego es crucial y se recomienda el uso de Serious Games o videojuegos comerciales en lugar de los denominados videojuegos educativos.

Prensky (2001), es uno de los principales promotores de dicha integración, iniciativa que ha llamado el "Digital Game Based Learning" es decir, el aprendizaje basado en juegos digitales. El autor propone que mediante el uso de videojuegos se podría favorecer el desarrollo de competencias como: la competitividad, la cooperación, la resolución de problemas, entre otras.

Trabajos como los de Gros (2009) y González Sánchez (2010), argumentan que la mayoría de los videojuegos educativos a la fecha de sus trabajos, han sido desarrollados poniendo mayor énfasis en el aspecto educativo que en el lúdico, perdiendo la eficacia por olvidar en su diseño la diversión e inmersión.

En esa línea, organizaciones como el New Media Consortium (https://www.nmc.org/) y el proyecto Serious Games (http://seriousgamesnet.eu/) discuten las estrategias para la aplicación de videojuegos, simulaciones, e hipermedia en contextos de aprendizaje (Davidson et. al., 2008). Poco a poco, las formas de aprendizaje intencional a través de juegos buscan una aproximación del concepto de diversión, por lo general como algo construido y no evidente, como lo sugiere la teoría del aprendizaje tangencial (Portnow, 2008). El aprendizaje tangencial es el proceso por el cual las personas se educan en un tema cuando se presenta en un contexto que disfrutan.

Aunque nuestra cultura establece una diferenciación muy grande entre lo lúdico y el aprendizaje como algo serio y formal. Gros (2009), Esnaola (2006) y Mayo (2009) destacan que existe cierta resistencia en los docentes en considerar a los videojuegos como herramientas para el aprendizaje, pues los consideran una parte de la actividad lúdica. Pero también algunos estudiantes tienen problemas para establecer la conexión entre el juego y el aprendizaje (Machargo et. al, 2003; Landivar y Magallanes (2006). En este sentido, es especialmente relevante la figura del profesor como experto en la determinación de los aprendizajes del juego.

Gee (2003), Gros (2009), Felicia (2009) y Prensky (2014) coinciden en afirmar que es importante conectar la experiencia del alumno como jugador con el punto de vista del aprendizaje.

Para ello es conveniente realizar una revisión y análisis de los eventos que ocurrieron en el juego en sí (Debriefing). La sesión de Debriefing proporciona un enlace entre lo que se representa en la 
simulación / juego con la experiencia y el mundo real. Permite al participante trazar paralelos entre los eventos del juego y los eventos del mundo real. Si nuestro interés estuviera en videojuegos "puros", este enlace no tendría que hacerse, ya que el juego existiría dentro de sus propios límites y se jugaría por sí mismo. Sin embargo, dado nuestro objetivo de trabajar con Serious Games, esta sesión de Debriefing nos permite transformar los eventos del juego en experiencias de aprendizaje. La sesión de Debriefing puede incluir una descripción de los eventos que ocurrieron en el juego, el análisis de porque ocurrieron y la discusión de errores y acciones correctivas (Garris, Ahlers \& Driskell, 2002).

Esta estrategia puede conducir a la abstracción de información relevante para que el aprendizaje efectivo ocurra y para que los estudiantes conecten el conocimiento adquirido al mundo real. Kolb, Rubin \& McIntyre (1971) calificaron este proceso de "hacer, reflexionar, comprender y aplicar". Es decir, las técnicas de evaluación y andamiaje proporcionan la orientación y el apoyo para ayudar a este proceso.

\section{Reflexiones finales}

Las nuevas generaciones han modificado sus formas de aprender, jugar y comunicar. La gestión del conocimiento está mediada con la tecnología y se generan nuevas conexiones todo el tiempo y en cualquier lugar.

Este panorama nos hace reflexionar acerca de los modelos enciclopedistas con los que se enseña en la actualidad. Un cambio de paradigma se avecina y en general no estamos preparados para enfrentarlo.

Las tecnologías emergentes acompañan estos avances culturales o podría considerarse que son parte de ello como respuesta a una demanda de la humanidad.

Entre estas tecnologías y prácticas de los jóvenes se ha abordado el caso de los videojuegos y en particular los Serious Games.

Los Serious Games pueden convertirse en una oportunidad de aprendizaje para el estudiante si el docente apuesta a utilizarlos, diseñando los distintos momentos de las sesiones de clase combinando su experiencia y las distintas propuestas de incorporación presentadas por las investigaciones en el campo.

El desafío está presente, depende de nosotros sacar provecho y explorarlo con una mentalidad abierta.

\section{Referencias Bibliográficas}

AARSETH, E. (1997). Cybertext. Perspectives on Ergodic Literature. Baltimore, The John Hopkins University Press.

ADAMS Becker, S., FREEMAN, A., Hall, C., CUMMINS, M., and YUHNKE, B. (2016). Reporte Horizont del NMC/CoSN: Edición 2016 K-12 Del Pre-Escolar al Grado 12. Austin, Texas: The New Media Consortium.

ALVAREZ, J. \& MICHAUD, L. (2008). Serious Games - Advergaming, edugaming, training and more. IDATE Consulting and Research.

ARANDA, D. y SÁNCHEZ Navarro J. (2009). “Algunas claves para entender los videojuegos”. En Aprovecha el tiempo y juega. Algunas claves para entender los videojuegos. Barcelona. Editorial UOC. pp. 7-36.

BECK, J. C. \& WADE, M. (2004). Got game: How the gamer generation is reshaping business forever. Boston: Harvard Business School Press. 
(2006). The Kids are alright: how the Gamer Generation is Changing the Workplace. Boston: Harvard Business School Press.

BOYLE, E., HAINEY T., CONNOLlY, T., GRAY, G., EARP, J., OTT, M., LIM, T., NINAUS, M., RIBEIRO, C. y PEREIRA, J. (2016). "An update to the systematic literature review of empirical evidence of the impacts and outcomes of computer games and Educational Games". En Computers \& Education, 94, pp. 178- 192.

CSIKSZENTMIHALYI, M. (1990). Flow: The psychology of optimal experience. New York: Harper and Row.

DAVIDSON, D., ALDRICH C., JACKSON M., EGENFELDT-NIELSEN S., THOMAS D. \& LEISHMAN D. (2008). Beyond Fun: Serious Games and Media. Pittsburgh: ETC Press.

DE FREITAS, S. \& LIAROKAPIS, F. (2011) Serious Games: A New Paradigm for Education?. M. Ma, et al. (Eds) Serious Games and Edutainment Applications (pp 9-23). UK: Springer.

DÍAZ-BARRIGA, A. (2014). "Competencias. Tensión entre programa político y proyecto educativo." En Propuesta Educativa, 42(23), 9- 27.

ESNAOLA, G. (2006). Claves culturales en la construccion del conocimiento. ¿Que enseñan los videojuegos? Buenos Aires: Alfagrama.

FELICIA, P. (2009). Videojuegos en el aula: manual para docentes. Bruselas: European Schoolnet.

FOREMAN, J. (2004). Game-Based Learning: How to Delight and Instruct in the 21st Century. EDUCAUSE Review.

FRASCA, G. (2009). “Juego, videojuego y creación de sentido. Una introducción” En Comunicación, 7, 37-44.

FULLAN, M. (2007). Las fuerzas del cambio con creces. Madrid: Akal

GARCÍA Gigante, B. (2009). Videojuegos: medio de ocio, cultura popular y recurso didáctico para la enseñanza y aprendizaje de las matemáticas escolares. Tesis Doctoral. Madrid: Facultad de Formación de Profesorado y Educación de la Universidad Autónoma de Madrid.

GARRIS, R., AHLERS, R., \& DRISKELL, J. E. (2002). "Games, motivation. And learning: a research and practice model.” En Simulation \& Gaming, 33(4), 441-467.

GEE, J. P. (2003). What Digital Games Have to Teach Us About Learning and Literacy. New York \& Basingstoke: Palgrave Macmillan.

GONZÁLEZ SÁNCHEZ, J. L. (2010). Jugabilidad. Caracterización de la experiencia del jugador en videojuegos. Tesis Doctoral. Universidad de Granada.

GROS, B. (2009). Certezas e interrogantes acerca del uso de los videojuegos para el aprendizaje. Comunicación, 7(1), 251-264.

HOWE, N. y STRAUSS, W. (2000). Millennials Rising: The Next Great Generation. New York: Vintage Original.

HUANG, W. D., JOHNSON, T. E., \& HAN, S. H. C. (2013). Impact of online instructional game features on college students' perceived motivational support and cognitive investment: a structural equation modeling study. The Internet and Higher Education, 17, 58-68.

KOSTER, R. (2013). Theory of Fun for Game Design. 2nd Edition. O'Reilly Media 
KOLB, D. A., RUBIN, I. M. \& MCINTYRE. J. (Eds.). (1971). Organizational psychology: An experiential approach. Englewood Cliffs, NJ: Prentice Hall.

LANDIVAR, T. y MAGALLANES, S. (2006). “Alumnos, docentes y videojuegos”. En I Congreso de Tecnología en Educación y Educación en Tecnología (TEYET 2006). REDUNCI.

MACHARGO, J., LIJAN, I., LEÓN, M., LÓPEZ, L. \& MARTIN, M., (2003). "Percepción de la influencia del ordenador, de Internet y de los videojuegos por los adolescentes". En Anuario de Filosofía, Psicología y Sociología, 6, 159-172.

MALONE, T. W. (1981). "Toward a theory of intrinsically motivating instruction". En Cognitive Science, 5, 333-369.

MAYO, M. (2009). “Video games: A route to large -scale STEM education?” En Science, 323(5910), 79-82. doi:10.1126/science.1166900

MEDINA, A. (2001). "Los métodos en la enseñanza universitária”. En Didáctica universitária Madrid: La Muralla. pp. 155-198

PADILLA ZEA, N. (2011). Metodología para el diseño de videojuegos educativos sobre una arquitectura para el análisis del aprendizaje colaborativo. Tesis doctoral. Universidad de Granada.

PÉREZ Latorre, Ó. (2012). El lenguaje videolúdico. Análisis de la significación del videojuego. España. Editorial Laertes.

PORTNOW, J. (2008). The power of tangential learning. Recuperado de http://www.edgeonline. com/blogs/the-power-tangential-learning.

PRENSKY, M. (2001). Digital game-based learning. New York: McGraw-Hill.

---, (2014). "Enseñar y formar en el tercer milenio". Conferencia impartida en la SIMO Educación 2014 (Salón de Tecnología para la Enseñanza). Madrid: España.

RAMÍREZ, M. (2014). Tecnologías emergentes en el movimento educativo abierto. Monterrey, México: Tecnológico de Monterrey.

SCOLARI, C. (2013). Homo videoludens 2.0. De Pacman a la gamification, Laboratori de Mitjans Interactius. Universitat de Barcelona, Barcelona.

SHERNOFF, D. J., CSIKSZENTMIHALYI, M., SCHNEIDER, B. \& SHERNOFF, E. S. (2003)."Student engagement in high school classrooms from the perspective of flow theory". En School Psychology Quarterly, 18 (2), 158-176.

SHERRY, J. L. (2013). "The challenge of audience reception: A developmental model for educational game engagement". En, Digital Games: A Context for Cognitive Development, 139, (pp. 11-20) 139, $11-20$.

SQUIRE, K. (2006). From Content to Context: Videogames as Designed Experience. Educational Researcher.

STEINER, C. M., HOLLINS, P., KLUIJFHOUT, E., DASCALU, M., NUSSBAUMER, A., ALBERT, D., \& WESTERA, W. (2015). "Evaluation of serious games: A holistic approach". International Conference of Education, Research and Innovation (ICERI 2015). España.

TAPSCOTT, D. (1998). Growing up digital. The rise of the net generation. New York: McGraw-Hill. 
ULICSAK, M. (2010). Games in Education: Serious Games-A Futurelab Literature Review. http://media.futurelab.org.uk/resources/documents/lit_reviews/Serious-Games_Review.pdf

VALDERRAMA, J. A. (2012). “Los videojuegos: conectar alumnos para aprender”. En Sinéctica, 39.

VOGEL, J. J., VOGEL, D. S., CANNON-BOWERS, J., BOWERS, C. A., MUSE, K. \& WRIGHT, M. (2006). "Computer gaming and interactive simulations for learning: A meta-analysis". En Journal of Educational Computing Research, 34, 229 -243.

YANG, Y. T. C. (2012). Building virtual cities, inspiring intelligent citizens: digital games for developing students' problem solving and learning motivation. Computers \& Education, 59(2), 365377.

ZYDA, M. (2005). "From visual simulation to virtual reality to games”. En Computer, 38(9), 25-32. 